\title{
Another proof of the Shirali-Ford theorem
}

\author{
Michael Leinert
}

\begin{abstract}
Shirali and Ford showed that every hermitian Banach $*$-algebra is symmetric. Meanwhile there have been several proofs of this theorem. We give another proof, a fairly conceptual one. It actually shows that every $*$-algebra which admits a spectral $C^{*}$-seminorm is completely symmetric.
\end{abstract}

Let $A$ be a Banach *-algebra, i.e. a Banach algebra with a (not necessarily continuous) involution. Suppose that $A$ is hermitian, i.e. the spectrum of selfadjoint elements $a=a^{*} \in A$ is real. The famous theorem of Shirali and Ford then states that $A$ is symmetric, i.e. every element $a^{*} a$, where $a \in A$, has its spectrum $\sigma\left(a^{*} a\right)$ contained in $[0, \infty)$. There are several proofs of this theorem (see [DB; Theorem 33.2] and the comments before it as well as [Pt], [B1], [B2], [F]). We give another proof starting from the fact that, on hermitian algebras, the Pták functional $s(a)=r\left(a^{*} a\right)^{1 / 2}$, where $r(b)$ denotes the spectral radius of $b \in A$, is a spectral $C^{*}$-seminorm, i.e. a $C^{*}$-seminorm which dominates the spectral radius on $A$ (see $\left[\mathrm{DB}\right.$; Theorem 33.1(a), (d), (j), (k)] for a proof of this fact). Let $A_{1}$ denote the algebra with unit adjoined.

THEOREM (Shirali and Ford). Every hermitian Banach *-algebra is symmetric.

Proof. $A$ is hermitian if and only if $A_{1}$ is hermitian, as is easily checked, so we may suppose $1 \in A$. Let $a \in A$ and $\lambda \in \sigma_{A}\left(a^{*} a\right)$. If $B$ is the closed subalgebra of $A$ generated by 1 and $a^{*} a$, by Gelfand's theorem there is an algebra homomorphism $\varphi: B \rightarrow \mathbb{C}$ with $\varphi\left(a^{*} a\right)=\lambda$. For $b \in B$ we have $|\varphi(b)| \leq r_{B}(b)=r_{A}(b) \leq s(b)$, the last inequality holding because $A$ is hermitian ([DB; 33.1(a)]). Since $s$ is a seminorm on $A$, by Hahn-Banach there is a linear extension $f: A \rightarrow \mathbb{C}$ of $\varphi$ with $|f(c)| \leq s(c)$ for all $c \in A$. Since $f(1)=\varphi(1)=1$, the following proposition implies positivity of $f$, hence $\lambda=\varphi\left(a^{*} a\right)=f\left(a^{*} a\right) \geq 0$.

Proposition. Let $q$ be a $C^{*}$-seminorm on a complex $*$-algebra $A$ with unit. Let $f: A \rightarrow \mathbb{C}$ be linear with $|f(a)| \leq q(a)$ for all $a \in A$ and $f(1)=1$. Then $f$ is positive.

Proof. For a $C^{*}$-algebra this is well known (see for instance [DB; Corollary $22.18]$ ). So the proof is a reduction to this case. For the reader's convenience, we

2000 Mathematics Subject Classification. MSC: 46 K 05.

Key words and phrases. Hermitian algebra, symmetric algebra, Shirali-Ford theorem. 
write the argument down. The set $N=\{a \in A \mid q(a)=0\}$ is $a$ *-ideal in $A$. On the $*$-algebra $A / N$ define $\dot{f}$ and $\dot{q}$ by $\dot{f}(\dot{a})=f(a), \dot{q}(\dot{a})=q(a)$ where $\dot{a}=a+N$, $a \in A$. Then $\dot{q}$ is a $C^{*}$-norm on $A / N$, and $|\dot{f}(\dot{a})| \leq \dot{q}(\dot{a})$ for $\dot{a} \in A / N$. Denote the completion of $(A / N, \dot{q})$ by $(C,\|\|)$, and the continuous extension of $\dot{f}$ to $C$ by $F$. We have $|F(b)| \leq\|b\|$ for $b \in C$ and $F(i)=1$, hence $\|F\|=F(i)$ where $i$ is the unit of $C$. Since $C$ is a $C^{*}$-algebra, $F$ is positive. So, for $a \in A$, we have $f\left(a^{*} a\right)=F\left(\dot{a}^{*} \dot{a}\right) \geq 0$, i.e. $f$ is positive.

REMARK 1. If we replace $a^{*} a$ in the proof of the theorem by $a_{1}^{*} a_{1}+\ldots+a_{k}^{*} a_{k}$, we obtain complete symmetry of $A$ (i.e. the spectrum of elements $a_{1}^{*} a_{1}+\ldots+a_{k}^{*} a_{k}$ is contained in $[0, \infty))$. The concept of complete symmetry is due to Wichmann [W].

REMARK 2. If one wants to use a more elementary argument (without use of Gelfand's theorem), the third and fourth sentence of the theorem's proof should be replaced by "The map $\varphi: \sum_{0}^{n} \alpha_{k}\left(a^{*} a\right)^{k} \mapsto \sum_{0}^{n} \alpha_{k} \lambda^{k}$ from the subalgebra $B$ of all polynomials in $a^{*} a$ to the complex numbers $\mathbb{C}$ is well defined, linear, and satisfies $|\varphi(b)| \leq r_{A}(b) \leq s(b)$ for $b \in B$. The last inequality holds because $A$ is hermitian."

From the above remarks and the theorem's proof we obtain the following

COROLlary. Every complex *-algebra which admits a spectral $C^{*}$-seminorm is completely symmetric.

Proof. (i) If $A$ is a $*$-algebra with unit, $q$ a spectral $C^{*}$-seminorm on it, let $x_{1}, \ldots, x_{n} \in A$ and $y=\sum x_{i}^{*} x_{i}$. If $\lambda \in \sigma(y)$, the map $\varphi: p(y) \mapsto p(\lambda)$ is well defined linear from the subalgebra $B$ of all polynomials in $y$ to $\mathbb{C}$ satisfying $|\varphi(b)| \leq r(b) \leq q(b)$ for all $b \in B$. By Hahn-Banach there is a linear extension $f: A \rightarrow \mathbb{C}$ with $|f(a)| \leq q(a)$ for all $a \in A$. Since $f(1)=\varphi(1)=1, f$ is positive (see the Proposition), so $\lambda=\varphi(y)=f(y)=f\left(\sum x_{i}^{*} x_{i}\right) \geq 0$.

(ii) If $A$ has no unit, let $r_{1}$ denote the spectral radius in $A_{1}, q_{1}$ the canonical $C^{*}$ seminorm extension of $q$ to $A_{1}$. Since $(\mu+a) \mapsto|\mu|$ is a $C^{*}$-seminorm on $A_{1}$, so is $q^{\prime}: \mu+a \mapsto \max \left\{|\mu|, q_{1}(a)\right\}$. For $\mu+a \in A_{1}$ one has $r_{1}(\mu+a) \leq|\mu|+r_{1}(a)=$ $|\mu|+r(a) \leq|\mu|+q(a) \leq 2 \max \left\{|\mu|, q_{1}(a)\right\}=2 q^{\prime}(\mu+a)$. For $c=\mu+a$ this implies $r_{1}(c)=r_{1}\left(c^{n}\right)^{1 / n} \leq 2^{1 / n} q^{\prime}(n) \rightarrow q^{\prime}(n)$, so $q^{\prime}$ is a spectral seminorm on $A_{1}$. By $(\mathrm{i})$, $A_{1}$ and hence $A$ is completely symmetric.

D.Birbas states in [B1, Theorem 3.2(i)] that every involutive algebra with realvalued subadditive Pták function (which then is a spectral $C^{*}$-seminorm, see [B1,Lemma 3.1]) is symmetric. His proof actually shows complete symmetry. At first sight, this seems to be a rather special case of the above Corollary, but on the other hand, any nonzero spectral $C^{*}$-seminorm has to coincide with the Pták function.

Let us also mention that the Corollary provides a more direct proof for the main part of [P,Proposition 10.4.2].

\section{References}

[B1] D. Birbas, Pták function and symmetry, Rend. Circ. Mat. Palermo, 47 (1998), pp. 431-446.

[B2] D. Birbas, On symmetric algebras, J. Math. Sciences 95 (1999), pp. 2609-2620.

[DB] R. S. Doran and V. A. Belfi, Characterizations of $C^{*}$-algebras. The Gel'fand-Naimark Theorems, Marcel-Dekker, New York and Basel, 1986. 
[F] M. Fragoulopoulou, The Shirali-Ford theorem as a consequende of Pták theory for hermitian Banach algebras, Studia Math. 150 (2002), pp. 121-132.

$[\mathrm{P}]$ Th. W. Palmer, Banach Algebras and the General Theory of *-Algebras, 2, Cambridge University Press, 2001.

[Pt] V. Pák, Banach algebras with involution, manuscr. math. 6 (1972), pp. 245-290.

[W] J. Wichmann, The symmetric radical of an algebra with involution, Arch. Math. 30 (1978), pp. $83-88$.

Institut für Angewandte Mathematik, Ruprecht-Karls-Universität Heidelberg, Im Neuenheimer Feld 294, D-69120 Heidelberg, Germany

E-mail address: leinert@math.uni-heidelberg.de 